\title{
Impact of current policies on future air quality and health outcomes in Delhi, India
}

Hem Dholakia (1), Pallav Purohit (2), Shilpa Rao (2), and Amit Garg (1)

(1) Public Systems Group, Indian Institute of Management, Ahmedabad - 380015, India, (2) International Institute for Applied Systems Analysis, Laxenburg, Austria

Mitigating adverse health impacts due to high concentrations of PM2.5 is a key policy challenge in Indian megacities. We carry out an integrated analysis of the air quality regulations across different sectors (transport, waste, power, industry) for the city of Delhi using the Greenhouse Gases and Air Pollution Interactions and Synergies (GAINS) model. We find that recommended national ambient air quality standards (NAAQS) for PM2.5 concentrations will not be met even by 2030 under the current policies scenario. Adopting advanced control technologies reduces PM2.5 concentrations by about $60 \%$ by and all-cause mortality by half in 2030 . Climate-centric mitigation policies significantly reduce greenhouse gases, but show only a modest impact on reducing PM2.5 concentrations. Controlling the net flow of air pollution, especially from trans-boundary sources will play a crucial role in reducing pollution levels in Delhi city. Achieving NAAQ requires a stringent policy portfolio that combines advanced control technologies with a switch to cleaner fuels and the control of trans-boundary pollution. 